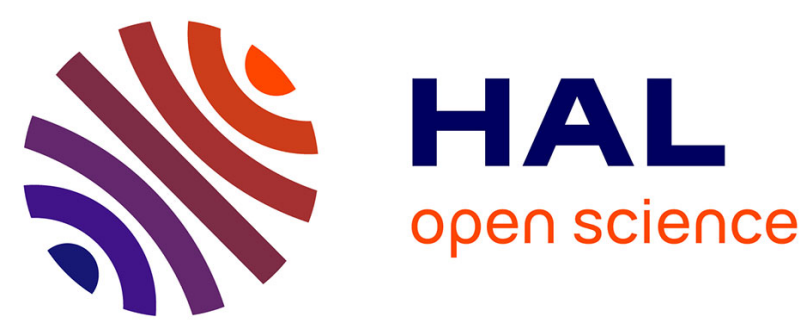

\title{
Non-linear vibrations of a beam with non-ideal boundary conditions and uncertainties - Modeling, numerical simulations and experiments
}

T Roncen, Jean-Jacques Sinou, J-P Lambelin

\section{- To cite this version:}

T Roncen, Jean-Jacques Sinou, J-P Lambelin. Non-linear vibrations of a beam with non-ideal boundary conditions and uncertainties - Modeling, numerical simulations and experiments. Mechanical Systems and Signal Processing, 2018, 110, pp.165-179. 10.1016/j.ymssp.2018.03.013 . hal-03257725

\author{
HAL Id: hal-03257725 \\ https://hal.science/hal-03257725
}

Submitted on 11 Jun 2021

HAL is a multi-disciplinary open access archive for the deposit and dissemination of scientific research documents, whether they are published or not. The documents may come from teaching and research institutions in France or abroad, or from public or private research centers.
L'archive ouverte pluridisciplinaire HAL, est destinée au dépôt et à la diffusion de documents scientifiques de niveau recherche, publiés ou non, émanant des établissements d'enseignement et de recherche français ou étrangers, des laboratoires publics ou privés. 
T. Roncen, J-J. Sinou and J-P. Lambelin, Non-linear vibrations of a beam with non-ideal boundary conditions and uncertainties - Modeling, numerical simulations and experiments, Mechanical Systems and Signal Processing, 110, 165-179, 2018.

doi.org/10.1016/j.ymssp.2018.03.013

\title{
on-linear vibrations of a beam with non-ideal boundary conditions and uncertainties - modeling, numerical simulations and experiments
}

\author{
T. Roncen ${ }^{\mathrm{a}, \mathrm{b}}$, J-J. Sinou ${ }^{\mathrm{a}, \mathrm{c}}$, J-P. Lambelin ${ }^{\mathrm{b}}$ \\ ${ }^{a}$ Laboratoire de Tribologie et Dynamique des Systèmes, UMR CNRS 5513, École Centrale de Lyon, 36 avenue Guy de \\ Collongue 69134 Écully Cedex, France \\ ${ }^{b}$ CEA, DAM, CESTA, F-33114 Le Barp, France \\ ${ }^{c}$ Institut Universitaire de France, 75005 Paris, France
}

\begin{abstract}
This paper presents experiments and numerical simulations of a nonlinear clamped-clamped beam subjected to Harmonic excitations and epistemic uncertainties. These uncertainties are propagated in order to calculate the dynamic response of the nonlinear structure via a coupling between the Harmonic Balance Method (HBM) and a non-intrusive Polynomial Chaos Expansion (PCE). The system studied is a clampedclamped steel beam.

First of all, increasing and decreasing swept sine experiments are performed in order to show the hardening effect in the vicinity of the primary resonance, and to extract the experimental multi-Harmonic frequency response of the structure. Secondly, the Harmonic Balance Method (HBM) is used alongside a continuation process to simulate the deterministic response of the nonlinear clamped-clamped beam. Good correlations were observed with the experiments, on the condition of updating the model for each excitation level. Finally, the effects of the epistemic uncertainties on the variability of the nonlinear response are investigated using a non-intrusive Polynomial Chaos Expansion (PCE) alongside the Harmonic Balance Method (HBM). A new methodology based on a phase criterion was developed in order to allow the PCE analysis to be performed despite the presence of bifurcations in the nonlinear response. The efficiency and robustness of the proposed methodology is demonstrated by comparison with Monte Carlo simulations. Then, the stochastic numerical results are shown to envelope the experimental responses for each excitation level without the need for model updating, validating the nonlinear stochastic methodology as a whole.
\end{abstract}

\section{Introduction}

The vibration response of mechanical systems used to be mainly studied by means of a linear analysis. Indeed, numerical simulations for both the modal analysis and frequency response function of linear systems are implemented in every Finite Element software package and are widely used in industry. However, experimentally, the dynamical system can experience nonlinear behavior with a frequency response that strongly depends on the excitation level. The nonlinearities may be caused by large displacements, contact, friction in the joints, or non-elastic compounds. The consequences of such nonlinearities are a dependency of eigenfrequencies and dissipation with input amplitude, discontinuities in the frequency response and a multi-Harmonic response to a mono-Harmonic excitation.

Both the efficient modeling of the nonlinear behavior of mechanical systems and the development of nonlinear computational techniques are essential in order to proceed with an efficient and quick analysis of complex problems. Various computational methods have been developed to compute the nonlinear differential equations, and used over a wide range of mechanical engineering problems $[1,2,3]$. Among these, the Incremental Harmonic Balance Method [4] enables the multi-Harmonic frequency response of a nonlinear system to be computed. This method was implemented in a previous work [5] and compared with the method of multiple scales and the shooting method for the computation of the nonlinear response of the mechanical system used in this paper. It was illustrated that the Harmonic Balance Method gave excellent results in terms of both precision and computation time.

Moreover, it is nowadays recognized that the effects of uncertainties on the nonlinear dynamics response of 
mechanical systems remain a major issue in engineering applications. Several approaches have been developed to estimate the stochastic dynamic response of mechanical systems: classical perturbation methods [6], the Monte-Carlo simulations [7], or Polynomial Chaos Expansion [8] for instance.

In the present study, we propose to investigate the nonlinear behavior of a clamped-clamped beam with large displacements, that is modeled by a Duffing oscillator. This system has been studied in the previous work by Claeys et al. [5] without considering uncertainties. This previous work brought to light the dispersion of the results with respect to the excitation level, and thus the need to take into account the uncertainties. Results from the deterministic nonlinear numerical simulations are compared with those from various experiments. The mandatory model updating for each experiment enforces the need for an epistemic uncertainties propagation strategy. These uncertainties are due to a lack of knowledge or incomplete information. The primary objective of the present study is the validation of an extended polynomial chaos expansion, in order to be able to predict the stochastic response of the beam despite the presence of bifurcations in the vicinity of the resonance.

The paper is divided into five parts. Firstly, a brief description of the test structure and the analysis of various experimental data from vibrational tests on a bench in the CEA laboratory are presented. Secondly, the paper focuses on the modeling of the beam. The nonlinear simulation based on the well-known Harmonic Balance Method and the non-intrusive polynomial chaos expansion procedure are detailed in Section 4 . Finally, results from the stochastic nonlinear numerical simulations are compared with those from a numerical reference (Monte-Carlo simulations) and the experiments. This last part illustrates the originality and peculiarities of the study, which proposes to build a non-intrusive polynomial chaos expansion for nonlinear vibrational systems with return points based on a phase criterion, leading to an increase in the confidence in the numerical models currently used with model uncertainties. The use of the phase criterion was proposed in previous works for linear [9] and nonlinear [10] systems subjected to uncertainties, using an intrusive approach and showing promising results.

\section{Experiments}

\subsection{Experimental setup}

The setup has been presented with minor changes relative to that presented in [5] for the same structure. The system studied is a steel beam of dimensions $470 \times 20 \times 5 \mathrm{~mm}$ presented in Figure 1 along with its instrumentation. The complete structure consists of the beam plus two heavy steel blocks of dimensions $100 \times 100 \times 85 \mathrm{~mm}$ each. The entire piece is manufactured from a single bulk piece of steel with a progressive link between the beam and the blocks in order to limit the stress concentrations in the clamped area.

The blocks are bolted onto a large circular aluminum plate, itself bolted onto the vibrating pot. The system is instrumented with 4 three-axial accelerometers. Two accelerometers ( $P 1$ and $P 2)$ are positioned on the top of the blocks, one is placed at the center of the beam $(A 1)$, and one is placed on the plate $(A 2)$. Each signal is denoted by the sensor name followed by the direction of measurement.

The entrance signal $A 2 Z$ is the shaker's feedback control signal. The reference frame of the study is the circular plate, where $A 2$ is fixed. The response signal is the acceleration at the middle of the beam, in the reference frame of the plate, that is $A 1 Z-A 2 Z$. The signals $P 1 Z$ and $P 2 Z$ help us to control the symmetry of the entrance signal.

\subsection{Experimental results and discussion}

The shaker is piloted with increasing and decreasing swept sines with a linear sweep rate. The excitation frequency evolves slowly over time $(0.1 \mathrm{~Hz} / \mathrm{s}$ in the vicinity of the resonance) to ensure that the response is stationary. Experimental results for three levels of excitation are plotted in Figure 2. The shape is typical of a Duffing oscillator.

The shaker was set to limit the lowest experimental excitation level to $1{\mathrm{~m} . \mathrm{s}^{-2}}$ because of the noise. The upper value was limited to $10 \mathrm{~m} . \mathrm{s}^{-2}$ in order to avoid the plasticizing of the beam. The experimental multiHarmonic input/output is presented in Figure 2 for a single level of excitation $\left(4 \mathrm{~m}^{-2} \mathrm{~s}^{-2}\right.$. The processing signal tool developed in [5] allows the identification of the fundamental frequency and the Harmonics of both the input and the output at every moment. The Harmonic identification is treated as a nonlinear optimization problem, using a Covariance Matrix Adaptation Evolution Strategy (CMAES, [11]). A concluding remark in the paper by Claeys et al. [5] is that the second output Harmonic originates from the input second Harmonic, while the third output Harmonic is due to the geometric nonlinearity in the vicinity of the resonance.

Some variations of the fundamental frequency of the beam (identified at low excitation) are observed from one experiment to another. In their work, Claeys et al. [5] assume that the frequency variations come from 


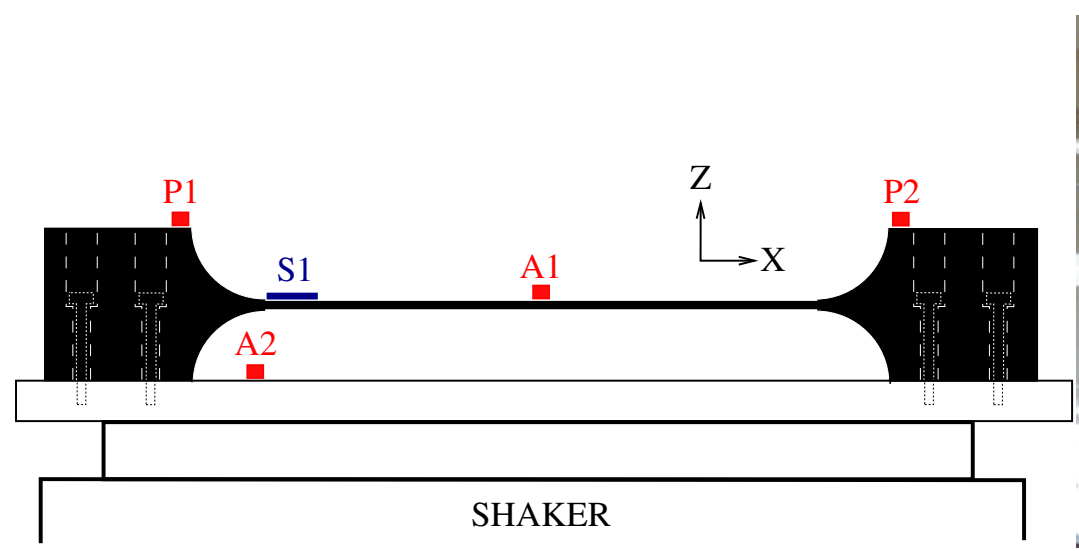

(a)

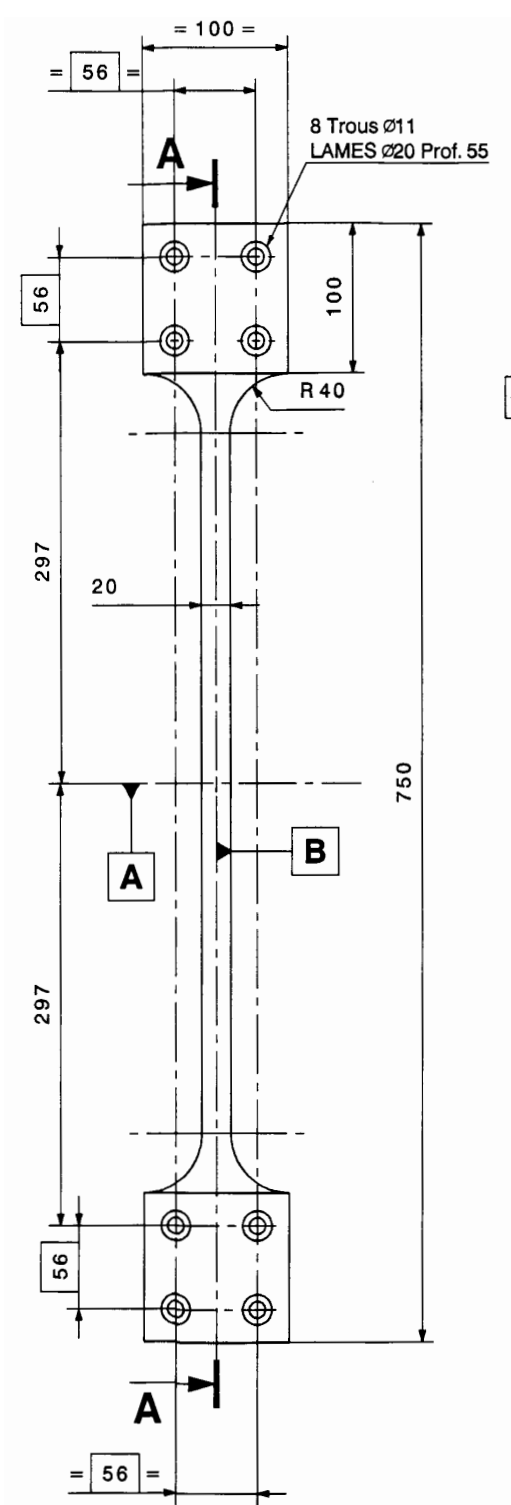

(c)

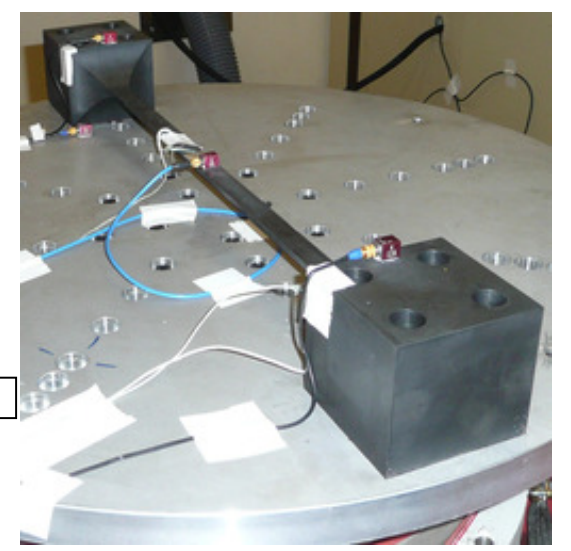

(b)

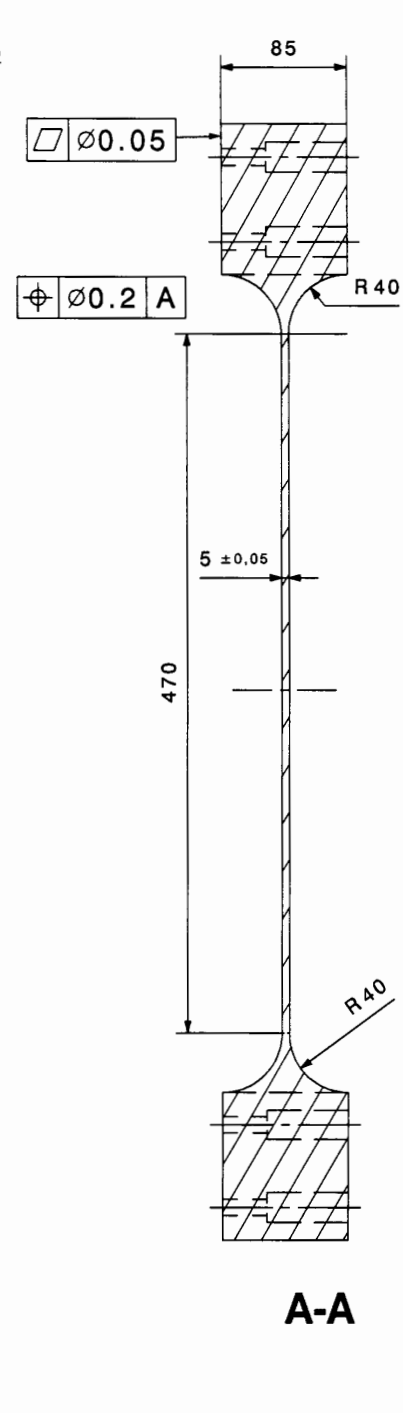

Figure 1: (a,b) Experimental setup (a,b) which includes 4 accelerometers (P1,P2,A1,A2) and geometry (c) of the beam 


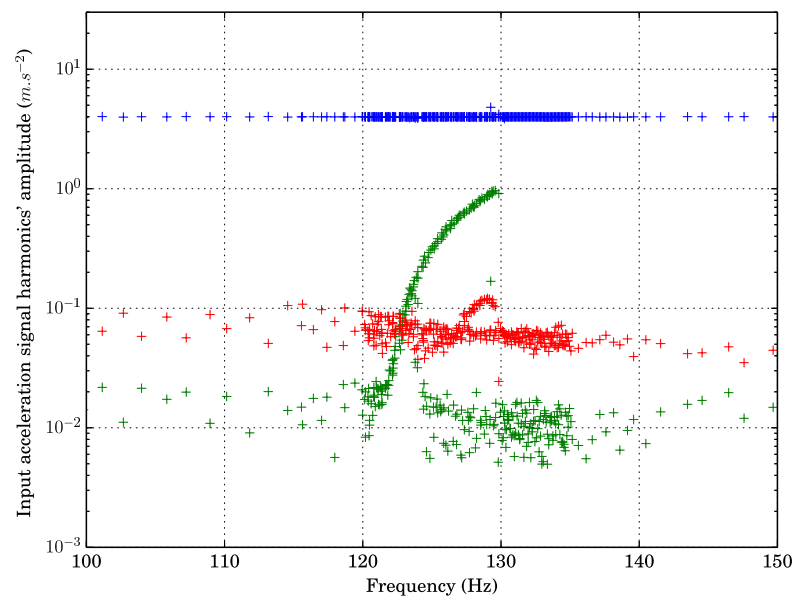

(a)

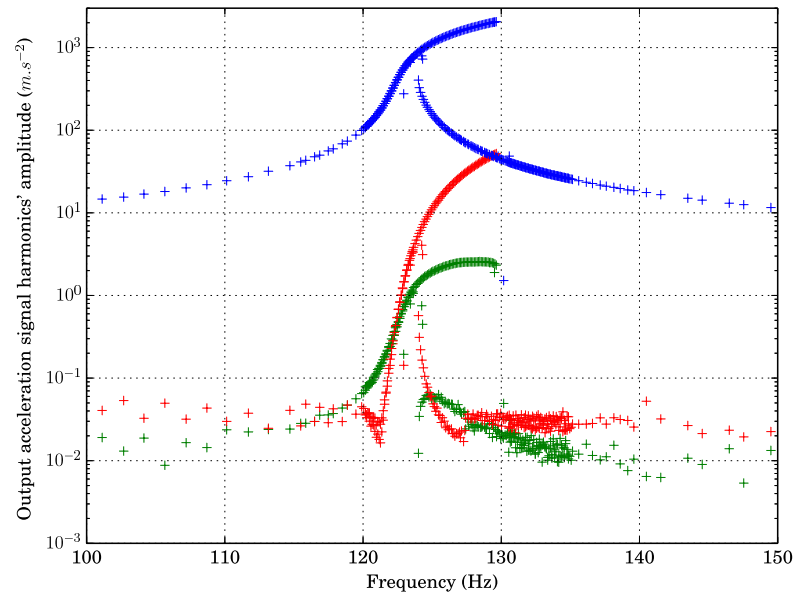

(b)

Figure 2: Input (a) and output (b) experimental Harmonics for the experiment with a constant excitation level of $4 \mathrm{~m} \cdot \mathrm{s}^{-2}$ for Harmonics 1 (blue), 2 (green) and 3 (red).

the static constraints in the beam, and they update the eigenfrequency through an effective half-length $(l)$ of the beam.

Regarding the resonance peak with hardening effect of the experimental response, which characterizes the nonlinear phenomena, it appears to be very reproducible from one experiment to another.

\section{Modeling}

\subsection{Modeling of the beam}

The notations are derived from the article [5]. We use the beam equation proposed by Nayfeh [12] to model the beam as a Duffing oscillator. We neglect the inertial and curvature nonlinear terms because of the small thickness-length ratio in our case, and we add a viscous damping coefficient $(\mu)$ to take into account the dissipation in the dynamical equation:

$$
\rho A \frac{\partial^{2} w}{\partial t^{2}}+\mu \frac{\partial w}{\partial t}+E I \frac{\partial^{4} w}{\partial x^{4}}=\rho A a_{e x c i t} \cos (\Omega t)+T(t) \frac{\partial^{2} w}{\partial x^{2}},
$$

where $\rho$ is the density $\left(\rho=7850 \mathrm{~kg} \cdot \mathrm{m}^{-3}\right), A$ is the beam section $\left(A=1.010^{-4} \mathrm{~m}^{2}\right), \mu$ is the viscous damping term, $E$ is Young's modulus $\left(E=20510^{9} \mathrm{~Pa}\right), I$ is the inertia momentum $\left(I=2.0810^{-10} \mathrm{~m}^{4}\right)$, $a_{\text {excit }}$ is the input vertical acceleration of the two blocks, $t$ is the time, $x$ is the x-axis, $w$ is the transverse displacement and $u$ is the axial displacement. $T(t)$ is the tensile strength in the beam. The tensile strength is invariant according to $x$ and satisfies the relationship:

$$
T(t)=E A\left(\frac{\partial u}{\partial x}+\frac{1}{2}\left(\frac{\partial w}{\partial x}\right)^{2}\right) \quad \forall x \in[0, l] .
$$

The boundary conditions are detailed in Equations(3) and (4). These boundary conditions take into account the imperfect boundary conditions. We therefore introduce two stiffness coefficients $k_{\text {rot }}$ and $k_{\text {bound }}$. The springs $k_{\text {rot }}$ and $k_{\text {bound }}$, the viscous damping coefficient $\mu$ and the half effective length $l$ are modeling parameters that will be updated in Section 5.1 and presented in Table 3 . The values of $k_{r o t}$ and $k_{\text {bound }}$ are fixed. It was shown by Claeys et al [5] that $k_{r o t}$ drives the modal participation factor (the $\Gamma_{i}$ introduced further in Equation (6)) while $k_{\text {bound }}$ drives the nonlinear curvature of the frequency response. Both the modal participation and the curvature remained constant throughout the experiments so these two variables will be updated once. However, the values of $\mu$ and $l$ will be updated throughout each experiment. These two variables are to be considered uncertain in Section 4.2 for the implementation of the Non-Intrusive Polynomial Chaos Expansion (NIPCE).

$$
\begin{gathered}
w(0)=0, \quad E I \frac{\partial^{3} w}{\partial x^{3}}(0)=k_{\text {rot }} \frac{\partial w}{\partial x}(0), \quad \frac{\partial w}{\partial x}(l)=0, \quad \frac{\partial^{3} w}{\partial x^{3}}(l)=0, \\
T(t)=k_{\text {bound }} u(0) \quad \text { et } \quad u(l)=0 .
\end{gathered}
$$


Considering Equations (1), (2),(3), (4), we build the nonlinear dynamical system as follows:

$$
\rho A \frac{\partial^{2} w}{\partial t^{2}}+\mu \frac{\partial w}{\partial t}+E I \frac{\partial^{4} w}{\partial x^{4}}=\rho A a_{\text {excit }} \cos (\Omega t)+\frac{E A}{2 l}\left(1+\frac{E A}{l k_{\text {bound }}}\right)^{-1}\left(\int_{0}^{l}\left(\frac{\partial w}{\partial x}\right)^{2} d x\right) \frac{\partial^{2} w}{\partial x^{2}} .
$$

Eqation (5) is then projected on the modal basis of its homogeneous equation in order to obtain a discrete equation whose Eigen vectors are $\mathbf{Y}_{\mathbf{i}}$ and the Eigen values are $\lambda_{i}$. The projection gives the discrete equation (demonstration can be seen in [12]):

$$
\frac{d^{2} w_{i}}{d t^{2}}+\frac{\mu}{\rho A} \frac{d w_{i}}{d t}+\Omega_{i}^{2} w_{i}=\Gamma_{i} a_{e x c i t} \cos (\Omega t)+\sum_{\{j, k, m\} \in M^{3}} \Gamma_{i j k m} \cdot w_{j} w_{k} w_{m} \quad \forall i \in M,
$$

where:

$$
\begin{gathered}
w_{i}(t)=\frac{Y_{i}(l)}{l} \int_{0}^{l} w(x, t) . Y_{i}(x) d x, \quad \Omega_{i}=\sqrt{\frac{E I}{\rho A}} \frac{\lambda_{i}^{2}}{l^{2}} ; \quad \Gamma_{i}=\frac{Y_{i}(l)}{l} \int_{0}^{l} Y_{i}(x) d x \\
\Gamma_{i j k m}=\frac{-E Y_{i}(l)}{2 \rho l^{2} Y_{j}(l) Y_{k}(l) Y_{m}(l)}\left(1+\frac{E A}{l k_{\text {bound }}}\right)^{-1}\left(\int_{0}^{l} \frac{d Y_{j}}{d x} \frac{d Y_{k}}{d x} d x\right)\left(\int_{0}^{l} \frac{d Y_{m}}{d x} \frac{d Y_{i}}{d x} d x\right) .
\end{gathered}
$$

It can be noted that the model chosen is a Duffing oscillator. This nonlinear model is responsible for the apparition of odd Harmonics in the numerical response, yet we observe both even and odd Harmonics in the experimental response. The even Harmonics are not predicted by the model, and cannot be explained by the multi-Harmonic input as demonstrated in [5]. For the rest of the article, we consider that the model is still a relevant approximation of the dynamical system around its first resonant mode.

\subsection{Modeling of the input excitation}

The numerical excitation is first a perfect sinusoid whose amplitude is equal to the experimental amplitude of the fundamental Harmonic. This purely sinusoidal numerical excitation allows simple calculations through HBM and the updating of the main parameters of the model. Section 5.1 shows the update of the parameters for different excitation levels.

Secondly, we build a numerical acceleration in which Harmonics 2 and 3 are constants and are equal to the maximum corresponding experimental values. An example of the numerical excitation is shown in Figure 3. This enriched input model will be used to estimate the numerical Harmonics in Section 5.

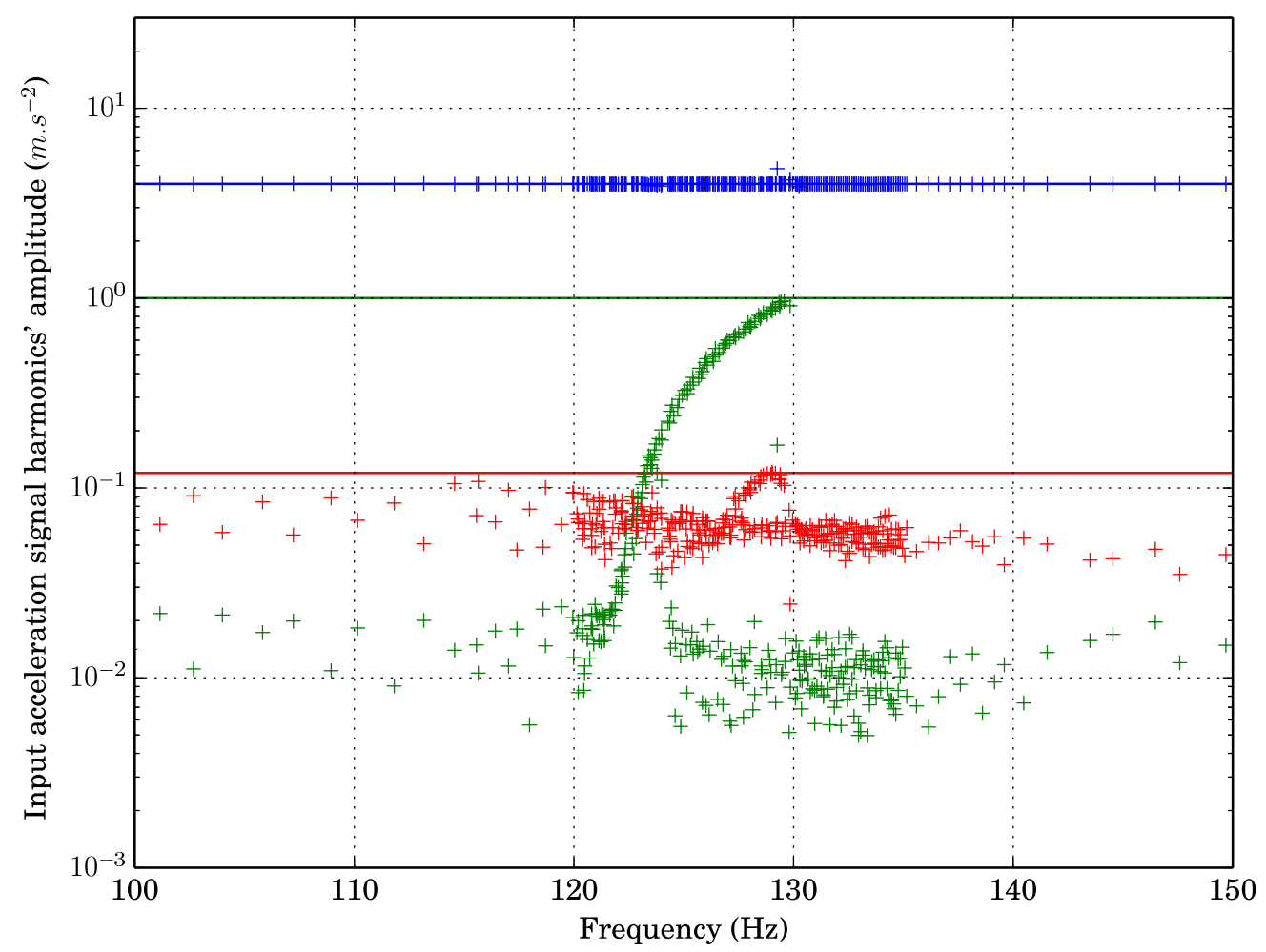

Figure 3: Experimental (cross) and numerical (solid lines) inputs for Harmonics 1 (blue), 2 (green) and 3 (red). 


\section{Nonlinear methodology}

In this section we develop the principle of both the Harmonic Balance Method and a Non-Intrusive Polynomial Chaos. These two methods are used adequately to obtain the stochastic response of the dynamic nonlinear problem.

\subsection{Deterministic non-linear problem}

A deterministic nonlinear dynamical system can be solved through an Harmonic Balance Method (HBM) [4]. To do so, the previous problem (6) is written in its equivalent matrix format:

$$
\ddot{\mathbf{W}}+\mathbf{D} \dot{\mathbf{W}}+\mathbf{K W}=\mathbf{F}_{\text {excit }}+\mathbf{F}_{\mathbf{n l}}(\mathbf{W}),
$$

where $\mathbf{W}$ is the vector of the eigenmodes of the beam, $\mathbf{D}$ is the modal damping, $\mathbf{K}$ is the modal stiffness, $\mathbf{F}_{\text {excit }}$ is the excitation force and $\mathbf{F}_{\mathbf{n l}}$ is the nonlinear term.

For a pulsation $\Omega$ of the excitation, the following equations are obtained:

$$
\begin{aligned}
\mathbf{W} & =\left\{w_{i}\right\}_{i \in M}, \quad \mathbf{D}=\operatorname{Diag}\left(\frac{\mu}{\rho A}\right)_{i \in M}, \quad \mathbf{K}=\operatorname{Diag}\left(\Omega_{i}^{2}\right)_{i \in M}, \\
\mathbf{F}_{\text {excit }} & =\left\{\Gamma_{i} a_{\text {excit }} \cos (\Omega t)\right\}_{i \in M}, \\
\mathbf{F}_{\mathbf{n l}}(\mathbf{W}) & =\left\{\sum_{\{j, k, m\} \in M^{3}} \Gamma_{i j k m} . w_{j} w_{k} w_{m}\right\}_{i \in M} .
\end{aligned}
$$

$M$ is the number of modes retained for the beam. Here we choose $M=1$ since we only study the first mode of the beam.

The nonlinear response $\mathbf{W}$, and the linear and nonlinear source terms (respectively $\mathbf{F}_{\text {excit }}$ and $\mathbf{F}_{\mathbf{n l}}(\mathbf{W})$ ) can be approximated by a truncated Fourier series up to an order $p . \quad\left(\mathbf{A}_{k}, \mathbf{B}_{k}\right)_{k \in[1, p]}, \quad\left(\mathbf{S}_{\mathbf{k}}, \mathbf{C}_{\mathbf{k}}\right)_{k \in[1, p]}$, $\left(\mathbf{S}_{\mathbf{k}, \mathbf{e x c i t}}, \mathbf{C}_{\mathbf{k}, \mathbf{e x c i t}}\right)_{k \in[1, p]}$ are the Fourier coefficients of the nonlinear response, and the nonlinear and linear source terms respectively:

$$
\begin{gathered}
\mathbf{W}(t)=\mathbf{B}_{\mathbf{0}}+\sum_{k=1}^{p}\left(\mathbf{B}_{\mathbf{k}} \cos (k \Omega t)+\mathbf{A}_{\mathbf{k}} \sin (k \Omega t)\right), \\
\mathbf{F}_{\mathbf{n l}}(t)=\mathbf{C}_{\mathbf{0}}+\sum_{k=1}^{p}\left(\mathbf{C}_{\mathbf{k}} \cos (k \Omega t)+\mathbf{S}_{\mathbf{k}} \sin (k \Omega t)\right), \\
\mathbf{F}_{\text {excit }}(t)=\sum_{k=1}^{p}\left(\mathbf{C}_{\mathbf{k}, \mathbf{e x c i t}} \cos (k \Omega t)+\mathbf{S}_{\mathbf{k}, \mathbf{e x c i t}} \sin (k \Omega t)\right),
\end{gathered}
$$

with:

$$
\mathbf{C}_{\mathbf{1}, \text { excit }}=\left\{\Gamma_{i} a_{e x c i t}\right\}_{i \in M}, \quad \mathbf{C}_{\mathbf{k}, \mathbf{e x c i t}}=0 \quad \forall k \neq 1, \quad \mathbf{S}_{\mathbf{k}, \mathbf{e x c i t}}=0 \quad \forall k \in[1, p] .
$$

The order $p$ of the Fourier series is selected on the basis of significant Harmonics expected in the nonlinear response. For this application, we choose $p=3$. The equation (7) is then written in the Fourier base:

$$
\left[\begin{array}{cc}
\mathbf{K}-(k \Omega)^{2} \mathbf{I} & -k \Omega \mathbf{D} \\
k \Omega \mathbf{D} & \mathbf{K}-(k \Omega)^{2} \mathbf{I}
\end{array}\right]\left[\begin{array}{l}
\mathbf{A}_{k} \\
\mathbf{B}_{k}
\end{array}\right]=\left[\begin{array}{l}
\mathbf{S}_{k} \\
\mathbf{C}_{k}
\end{array}\right]+\left[\begin{array}{c}
\mathbf{S}_{k, \text { excit }} \\
\mathbf{C}_{k, \text { excit }}
\end{array}\right] \quad \forall k \in[1, p],
$$

where $\mathbf{I}$ is the identity matrix. The coefficients $\mathbf{S}_{k}$ and $\mathbf{C}_{k}$ depend on the coefficients $\mathbf{A}_{\mathbf{k}}$ and $\mathbf{B}_{\mathbf{k}}$. They are calculated through an Alternating Frequency Time method (AFT,[13]). This method uses the Discrete Fourier Transform (DFT) to calculate the nonlinearity in the time domain before switching back to the Fourier base with an Inverse DFT (IDFT). The process is summed up in the following diagram:

$$
X=\left[\mathbf{B}_{\mathbf{0}} \mathbf{A}_{\mathbf{1}} \mathbf{B}_{\mathbf{1}} \ldots \mathbf{A}_{\mathbf{p}} \mathbf{B}_{\mathbf{p}}\right] \stackrel{D F T^{-1}}{\longrightarrow} W(t) \stackrel{E q \cdot 10}{\longrightarrow} F(t) \stackrel{D F T}{\longrightarrow}\left[\mathbf{C}_{\mathbf{0}} \mathbf{S}_{\mathbf{1}} \mathbf{C}_{\mathbf{1}} \ldots \mathbf{S}_{\mathbf{p}} \mathbf{C}_{\mathbf{p}}\right] .
$$

$X$ is the vector of the Fourier coefficients of the solution.

The latter equation creates a direct link between the Fourier coefficients of $\mathbf{W}$ and the coefficients of $\mathbf{F}_{\mathbf{n l}}$. This link enables a nonlinear resolution of the equation (15):

$$
\mathbf{G}(\mathbf{X}, \Omega)=\mathbf{0} .
$$

In order to compute a continuous response curve in the frequency-domain amplitude, a Moore-Penrose continuation is used. To implement the method, the fundamental pulsation $\Omega$ is considered as a variable 
of the problem along with the Fourier coefficients $\mathbf{X}$ of the response. Let $\mathbf{Y}=[\mathbf{X}, \Omega]$ be the continuation vector. The continuation consists of two steps: the prediction and the correction.

The prediction is the first step. The secant method is presented here and will be used in this work. We consider the two latest solution points found through the continuation method. Let $\mathbf{Y}_{\mathbf{n}-\mathbf{1}}$ and $\mathbf{Y}_{\mathbf{n}}$ be those points, where $\mathbf{Y}_{\mathbf{n}}=\left[\mathbf{X}^{(\mathbf{n})}, \Omega^{(n)}\right]$. We extrapolate the next point by following the direction of the secant line defined by the two points $\mathbf{Y}_{\mathbf{n}-\mathbf{1}}$ and $\mathbf{Y}_{\mathbf{n}}$. A parameter $\beta$ is introduced to control the distance of the new point:

$$
\left\|\mathbf{Y}_{\mathbf{n}+\mathbf{1}}^{\mathbf{0}}-\mathbf{Y}_{\mathbf{n}}\right\|=\beta\left\|\mathbf{Y}_{\mathbf{n}}-\mathbf{Y}_{\mathbf{n}-\mathbf{1}}\right\|
$$

The control parameter $\beta$ enables the speed convergence to be controlled. Typical values of $\beta$ are $1<\beta<2$ for weak nonlinear regions and $0.1<\beta<1$ for strong nonlinear regions. The distance between $\mathbf{Y}_{\mathbf{n}}$ and $\mathbf{Y}_{\mathbf{n}+\mathbf{1}}^{\mathbf{0}}$ is the step $\Delta s$ defined by:

$$
\mathbf{Y}_{\mathbf{n}+\mathbf{1}}^{\mathbf{0}}=\mathbf{Y}_{\mathbf{n}}+\Delta s .\left(\mathbf{Y}_{\mathbf{n}}-\mathbf{Y}_{\mathbf{n}-\mathbf{1}}\right) .
$$

Other types of predictions are detailed in [14].

The correction is the second step of the continuation. It is used to move the prediction closer to a zero of G. The Moore-Penrose correction is based on the generalized concept of the inverse matrix [15] in order to calculate the pseudo-inverse (noted with $\mathrm{a}+$ ) of the Jacobian of the optimization problem $\mathbf{G}(17)$ :

$$
\mathbf{J}_{\mathbf{y}}^{+} \mathbf{G}={ }^{\mathrm{t}} \mathbf{J}_{\mathbf{y}} \mathbf{G} \cdot\left(\mathbf{J}_{\mathbf{y}} \mathbf{G} \cdot{ }^{\mathrm{t}} \mathbf{J}_{\mathbf{y}} \mathbf{G}\right)^{-1} .
$$

A first order Taylor development gives:

$$
\mathbf{G}\left(\mathbf{Y}_{\mathbf{n}+\mathbf{1}}^{\mathbf{i}}\right)=\mathbf{G}\left(\mathbf{Y}_{\mathbf{n}+1}^{\mathbf{i}-1}\right)+\left(\mathbf{J}_{\mathbf{y}}^{+} \mathbf{G}\left(\mathbf{Y}_{\mathbf{n}+1}^{\mathbf{i}-1}\right)\right)^{-1} \times\left[\begin{array}{c}
\Delta \mathbf{X} \\
\Delta \Omega
\end{array}\right]
$$

The calculation of the Jacobian $\mathbf{J}_{\mathbf{y}} \mathbf{G}$ is made by finite differences where the two values of $G$ are evaluated using the AFT method.

At iteration $i, \mathbf{Y}_{\mathbf{n}+\mathbf{1}}^{\mathbf{i}}$ should be a $\mathbf{0}$ of $\mathbf{G}$, leading to:

$$
\mathbf{Y}_{\mathbf{n}+\mathbf{1}}^{\mathbf{i}}=\mathbf{Y}_{\mathbf{n}+\mathbf{1}}^{\mathbf{i}-1}+\left[\begin{array}{c}
\Delta \mathbf{X} \\
\Delta \Omega
\end{array}\right] \quad \text { with } \quad\left[\begin{array}{c}
\Delta \mathbf{X} \\
\Delta \Omega
\end{array}\right]=-\mathbf{J}_{\mathbf{Y}}^{+} \mathbf{G}\left(\mathbf{Y}_{\mathbf{n}+\mathbf{1}}^{(\mathbf{i}-\mathbf{1})}\right)^{-1} \mathbf{G}\left(\mathbf{Y}_{\mathbf{n}+\mathbf{1}}^{(\mathbf{i}-\mathbf{1})}\right)
$$

Once the Fourier coefficients $\mathbf{X}$ are calculated, the amplitude of Harmonic $k$ of $x$ is given by the formula $\mathbf{G}^{k}=\sqrt{\left\|\mathbf{A}_{k}\right\|^{2}+\left\|\mathbf{B}_{k}\right\|^{2}}$, so the frequency response can be calculated for each Harmonic.

In the end, the continuation enables the continuous solution curve to be found. This solution depends on the excitation and the physical parameters of the system. In Section 5.1, we show that two physical parameters of the model, $l$ and $\mu$, need to be updated. Let $l$ and $\mu$ be considered as uncertain parameters of the system. We will write $\mathbf{H}_{\mathbf{l} \mu}$ the function solution of Equation (17) for a given set of values for $l$ and $\mu$ :

$$
\forall \Omega, \mathbf{X}(\Omega)=\mathbf{H}_{\mathbf{l} \mu}(\Omega) .
$$

This simplified notation eludes the dependency of $\mathbf{H}_{1 \mu}$ on the other parameters of the system for the sake of clarity.

\begin{tabular}{cc}
\hline$p$ & $L_{k}(\xi)$ \\
\hline 0 & 1 \\
1 & $\xi$ \\
2 & $\frac{1}{2}\left(-1+3 \xi^{2}\right)$ \\
3 & $\frac{1}{2}\left(-3 \xi+5 \xi^{3}\right)$ \\
4 & $\frac{1}{8}\left(3-30 \xi^{2}+35 \xi^{4}\right)$ \\
5 & $\frac{1}{8}\left(15 \xi-75 \xi^{3}+63 \xi^{5}\right)$ \\
6 & $\frac{1}{16}\left(-5+105 \xi^{2}-315 \xi^{4}+231 \xi^{6}\right)$ \\
7 & $\frac{1}{16}\left(-35 \xi+315 \xi^{3}-693 \xi^{5}+429 \xi^{7}\right)$ \\
8 & $\frac{1}{128}\left(35-1260 \xi^{2}+6930 \xi^{4}-12012 \xi^{6}+6435 \xi^{8}\right)$ \\
9 & $\frac{1}{16}\left(315 \xi-4620 \xi^{3}+18018 \xi^{5}-25740 \xi^{7}+12155 \xi^{9}\right)$ \\
10 & $\frac{1}{256}\left(-63+3465 \xi^{2}-30030 \xi^{4}+90090 \xi^{6}-109395 \xi^{8}+46189 \xi^{10}\right)$ \\
\hline
\end{tabular}

Table 1: Legendre Polynomials for a uniform random variable $\xi$ 


\begin{tabular}{ccccc}
\hline Distribution & Fonction of repartition & Polynomial & Density $f_{X}(\xi)$ & Interval \\
\hline Normal & $\frac{1}{\sqrt{2 \pi}} e^{\frac{-\xi^{2}}{2}}$ & Hermite $H_{n}(\xi)$ & $e^{\frac{-\xi^{2}}{2}}$ & {$[-\infty, \infty]$} \\
Uniform & $\frac{1}{2}$ & Legendre $L_{n}(\xi)$ & 1 & {$[-1,1]$} \\
Exponential & $e^{-\xi}$ & Laguerre $L a_{n}(\xi)$ & $e^{-\xi}$ & {$[0, \infty]$} \\
\hline
\end{tabular}

Table 2: Hermite, Legendre and Laguerre polynomials associated to their stochastic distributions.

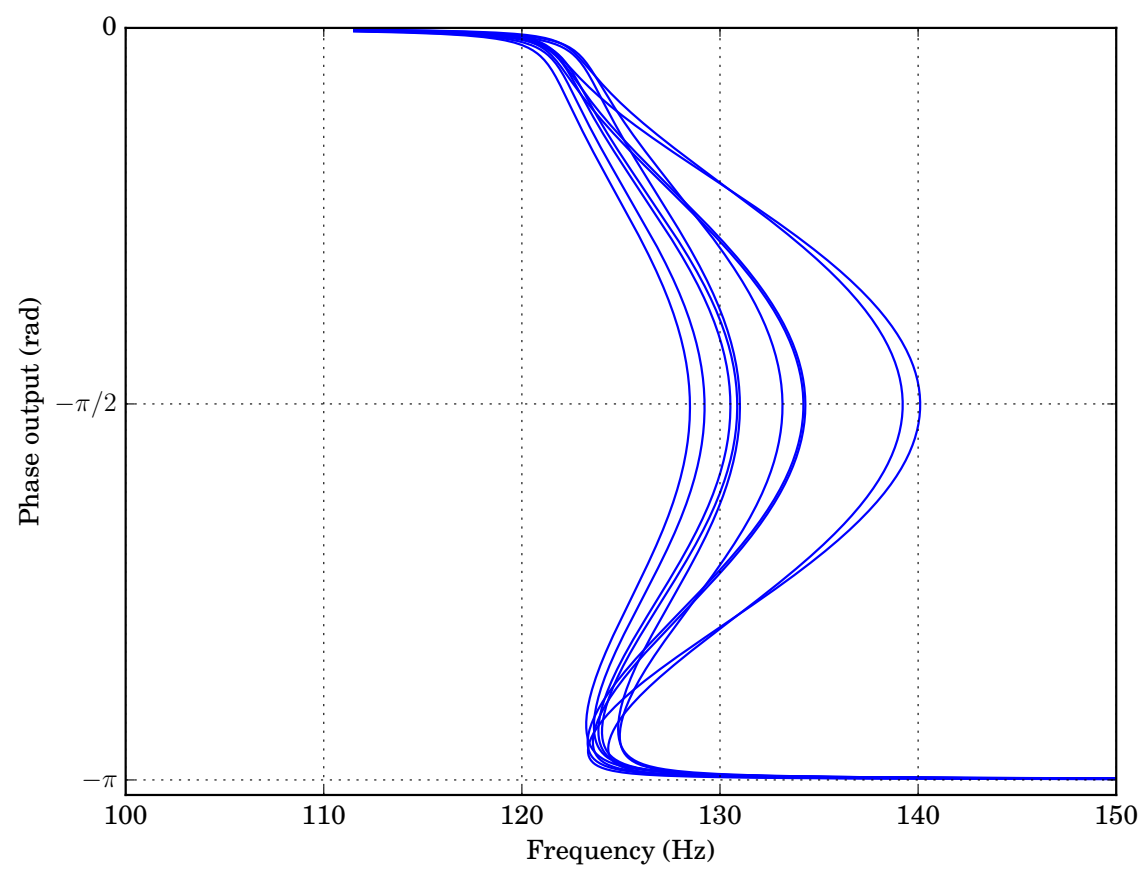

Figure 4: Phase evolution for different sets of deterministic numerical solutions.

\subsection{Stochastic non-linear problem}

When some input terms of a system are defined by a random distribution, the propagation of the uncertainties in the model can be studied using stochastic methods. The Monte-Carlo method is one of the first probabilistic methods used to propagate uncertainties, it will serve as a reference in this paper. Details of the method can be found in [7]. In order to solve nonlinear dynamical systems with uncertainties, previous studies $[16,17]$ proposed the use of a Stochastic-HBM combined with AFT and Probabilistic Collocation (AFTPC). These studies used an Intrusive Polynomial Chaos Expansion which has the drawback of being difficult to implement for larger structures or more complex physical problems. The Non-Intrusive Polynomial Chaos Expansion (NIPCE) allows the stochastic response of a system to be calculated with reduced computational efforts compared to Monte-Carlo methods without modification of the deterministic code, making it an interesting choice for the present study.

\subsubsection{Polynomial Chaos Expansion}

Polynomial chaos is a stochastic method based on the spectral representation of uncertainty. This domain has been studied by many researchers in recent years $[18,19,20,21]$ and is only a specific example of the more general Spectral Methods, detailed in [22]. An important aspect of the spectral representation of uncertainty is that a random function (or variable) can be decomposed into separable deterministic and stochastic components. For example, for a random field $\mathbf{H}(\mathbf{x}, \xi)$, we can write:

$$
\mathbf{H}(\mathbf{x}, \xi)=\sum_{i=0}^{P} \mathbf{H}_{i}(\mathbf{x}) \mathbf{\Psi}_{i}(\xi),
$$

where $\mathbf{H}_{i}(\mathbf{x})$ is the deterministic projection of $\mathbf{H}$ on the $i^{\text {th }}$ polynomial chaos and $\boldsymbol{\Psi}(\xi)$ is the polynomial basis. In theory the projection should include an infinite number of terms but we truncate the sum to an integer $P$. The number of terms in the equation $(24)$ is:

$$
N_{t}=P+1=\frac{(d+p) !}{d ! p !}
$$




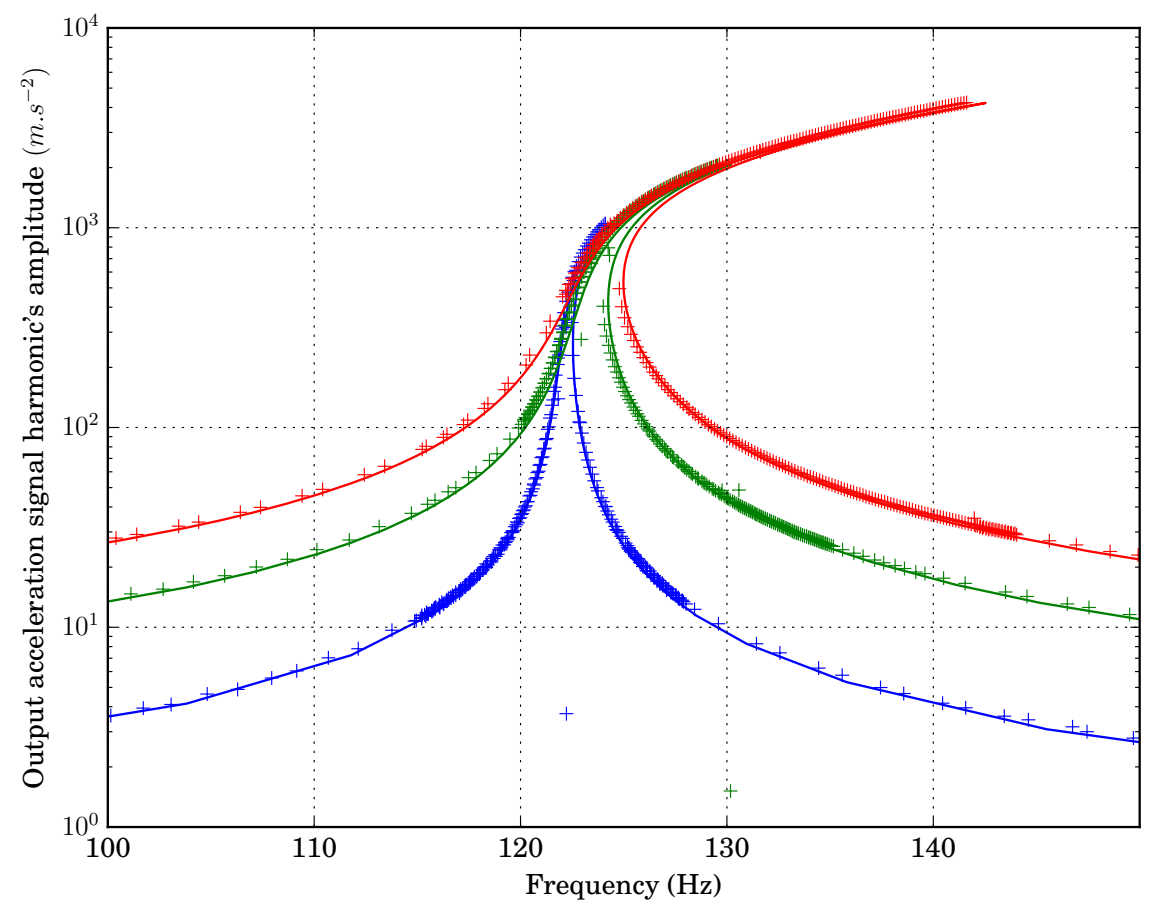

Figure 5: Fundamental harmonic of the experimental (crosses) and numerical (lines) responses. Excitation levels are 1 (blue), 4 (green) and 8 (red) $m . s^{2}$.

\begin{tabular}{clccc}
\hline & Parameter & Excit $=1 \mathrm{~m}^{-s^{-2}}$ & Excit $=4 \mathrm{~m} . \mathrm{s}^{-2}$ & Excit $=8 \mathrm{~m} . \mathrm{s}^{-2}$ \\
\hline $\mathrm{l}$ & Half effective length & $0.2357 \mathrm{~m}$ & $0.2348 \mathrm{~m}$ & $0.2345 \mathrm{~m}$ \\
$\mu$ & Damping coefficient & $0.7065 \mathrm{~kg} . \mathrm{m}^{2} . \mathrm{s}^{-1}$ & $1.492 \mathrm{~kg} \cdot \mathrm{m}^{2} . \mathrm{s}^{-1}$ & $1.609 \mathrm{~kg} . \mathrm{m}^{2} . \mathrm{s}^{-1}$ \\
$k_{\text {rot }}$ & Rotational stiffness & & $11.510^{4} \mathrm{~N} \cdot \mathrm{rad}^{-1}$ & \\
$k_{\text {bound }}$ & Axial stiffness & & $1.110^{8} \mathrm{~N} . \mathrm{m}^{-1}$ & \\
\hline
\end{tabular}

Table 3: Physical values of the updated parameters

where $d$ is the dimension of the random variable $\xi$ and $p$ is the polynomial chaos.

Multi-dimensional Hermite Polynomial was the first basis function to be used (See Wiener [23]) for polynomial chaos when the input was following a normal distribution. Many other choices are now possible thanks to a later development by Xiu and Karniadakis [20], which extended the notion to other kinds of variables (uniform, exponential, etc.). Table 2 shows the usual orthogonal polynomial families used for a chosen stochastic distribution. Table 1 gives the first Legendre polynomials for a unidimensional random variable $\xi$ up to the order $p=10$. The objective of the stochastic methods based on polynomial chaos is to determine the coefficient of each term $\left(\mathbf{H}_{i}(\mathbf{x}), i=0 . . P\right)$ in the polynomial expansion given in 24 .

Two commonly used approaches for non-intrusive polynomial chaos are sampling-based and quadrature methods. In order to find the polynomial coefficient $\mathbf{H}_{k}(\mathbf{x})$ in Equation 24, the equation is projected onto the $k^{t h}$ basis:

$$
\left\langle\mathbf{H}(\mathbf{x}, \xi), \mathbf{\Psi}_{k}(\xi)\right\rangle=\left\langle\sum_{i=0}^{P} \mathbf{H}_{i}(\mathbf{x}) \mathbf{\Psi}_{i}(\xi), \mathbf{\Psi}_{k}(\xi)\right\rangle
$$

The orthogonality of the polynomial basis leads to:

$$
\left\langle\mathbf{H}(\mathbf{x}, \xi), \mathbf{\Psi}_{k}(\xi)\right\rangle=\mathbf{H}_{k}(\mathbf{x})\left\langle\mathbf{\Psi}_{k}(\xi)^{2}\right\rangle
$$

Finally, we obtain:

$$
\mathbf{H}_{k}(\mathbf{x})=\frac{\left\langle\mathbf{H}(\mathbf{x}, \xi), \mathbf{\Psi}_{k}(\xi)\right\rangle}{\left\langle\mathbf{\Psi}_{k}(\xi)^{2}\right\rangle}
$$

In sampling-based methods, the main strategy is to compute $\left\langle\mathbf{H}(\mathbf{x}, \xi), \mathbf{\Psi}_{k}(\xi)\right\rangle$ for a number of samples and to perform the average, in order to determine the estimate of the inner product $\left\langle\mathbf{H}(\mathbf{x}, \xi), \Psi_{k}(\xi)\right\rangle$. The quadrature method calculates the same term but through a Gauss-Hermite Quadrature point procedure. In the end, both methods use Equation (28) to estimate the projected polynomial coefficients. 


\subsubsection{Description of the Present Non-Intrusive Polynomial Chaos Method}

The present polynomial chaos uses a point-collocation method as a viable and simple alternative to sampling-based and quadrature-based methods. This approach was first used by Walters [24] and then used successfully for various applications $[25,26]$.

We build a family of $N>P+1$ vectors $\left(\xi_{\mathbf{i}}=\left(\xi_{1}, \xi_{2}, . ., \xi_{d}\right)_{i}, i=0,1, \ldots, N\right)$ where every member of the family is a realization of the random vector $\xi$. The $\xi_{\mathbf{i}}$ are chosen adequately in the space of the random variables by using a Latin Hypercube Sampling (LHS, see [27] for details).

A deterministic evaluation of the nonlinear dynamical code is realized for each $\xi_{\mathbf{i}}$ using the deterministic HBM, which makes the $N \mathbf{H}\left(\mathbf{x}, \xi_{\mathbf{i}}\right)$. Using Equation (24), the following linear system is obtained:

$$
\left[\begin{array}{cccc}
\boldsymbol{\Psi}_{0}\left(\xi_{\mathbf{0}}\right) & \boldsymbol{\Psi}_{1}\left(\xi_{\mathbf{0}}\right) & \cdots & \boldsymbol{\Psi}_{P}\left(\xi_{\mathbf{0}}\right) \\
\boldsymbol{\Psi}_{0}\left(\xi_{\mathbf{1}}\right) & \boldsymbol{\Psi}_{1}\left(\xi_{\mathbf{1}}\right) & \cdots & \boldsymbol{\Psi}_{P}\left(\xi_{\mathbf{1}}\right) \\
\vdots & \vdots & \ddots & \vdots \\
\boldsymbol{\Psi}_{0}\left(\xi_{\mathbf{N}}\right) & \boldsymbol{\Psi}_{1}\left(\xi_{\mathbf{N}}\right) & \cdots & \boldsymbol{\Psi}_{P}\left(\xi_{\mathbf{N}}\right)
\end{array}\right]\left[\begin{array}{c}
\mathbf{H}_{0}(\mathbf{x}) \\
\mathbf{H}_{1}(\mathbf{x}) \\
\vdots \\
\mathbf{H}_{P}(\mathbf{x})
\end{array}\right]=\left[\begin{array}{c}
\mathbf{H}\left(\mathbf{x}, \xi_{\mathbf{0}}\right) \\
\mathbf{H}\left(\mathbf{x}, \xi_{\mathbf{1}}\right) \\
\vdots \\
\mathbf{H}\left(\mathbf{x}, \xi_{\mathbf{N}}\right)
\end{array}\right]
$$

The coefficients $\mathbf{H}_{\mathbf{i}}(\mathbf{x})$ are obtained through the resolution of the linear system. The method described here is non-intrusive, since there is no modification of the deterministic code. It is shown that a number $N>2 P$ points (i.e., $N$ HBM) coupled to the LHS yields excellent results. It was shown in [26] that even though both point-collocation and quadrature methods suffer from the curse of dimensionality, the point-collocation with LHS scales better than a classic Smolyak-grid quadrature approach for large scale problems. In our application though, the difference between the two methods would be small.

\subsubsection{Linking of the HBM and the NIPC}

Two problems emerge from the study of the stochastic response of a nonlinear dynamical system with bifurcations. Firstly, the response frequency of a linear vibration problem is always deterministic for a given set of frequency and random variables. This is no longer the case for a nonlinear system with return points, where a frequency can give three different deterministic solutions in the vicinity of the response. Therefore, the position of the return points will depend on the random variables, leading to a stochastic behavior of the frequency. The NIPC can then no longer be used to determine the stochastic response in the frequency domain amplitude. The second problem arises from the discontinuity of the nonlinear response over the frequency domain. The discontinuity cannot be approached by a polynomial, unless the polynomial degree is not extremely high [28]. This leads to spurious oscillations, called the Gibbs phenomenon, which deteriorate the quality of the frequency response functions.

These two problems have been encountered and dealt with in a recent article by Panunzio et al [29]. They used an Asymptotic Numerical Method (ANM, see [30] for details) coupled with an NIPCE for a Duffing oscillator. The authors introduced a parameter called the "arc-length-ratio" to replace the frequency in the making of the NIPCE. This "arc-length-ratio" ensures that the critical points (the minima, maxima and return points) of the response are not dependent on the values of the random variables. In order to work properly, the critical points need to be detected during the ANM, and the response curve is divided into solution branches defined between two critical points. There are as many arc-length-ratios as branches, and they all go from 0 (beginning of the branch) to 1 (end of the branch). This ensures the deterministic behavior of the parameter and the disappearance of the Gibbs phenomenon.

In this paper, we use an HBM without ANM and the definition of the arc-length is not as straightforward. Rather than updating the deterministic code, we propose to use another parameter to replace the arc-lengthratio: the phase. One of the advantages of this choice is that we use a criterion based on physical observation in order to gather different points from all of the response curves.

The phase always refers to the phase angle between the fundamental of the output acceleration and the fundamental of the input acceleration. The evolution of the phase with respect to the frequency is presented in Figure 4 for different realizations of uncertain variables $l$ and $\mu$ with a constant excitation level of $4 \mathrm{~m}_{\mathrm{s}} \mathrm{s}^{-2}$. Each realization requires an HBM process to be performed. We observe that the phase is always a strictly decreasing function over the deterministic HBM calculation. Therefore, the amplitude and the frequency do not have bifurcations with respect to the phase for a given deterministic calculation. This makes the phase a good candidate for being the deterministic parameter of an NIPCE. The use of the phase has two advantages compared to an arc-length-ratio. Firstly, as previously explained, it is a physical parameter that engineers are familiar with: the phase is of strong importance in experimental dynamics. Secondly, it is not necessary to split the numerical solution into different branches, since the phase is already almost the same for the critical points. It can be seen in Figure 4 that the critical points are obtained almost at the same phase for every realization. This behavior will impede the Gibbs phenomenon. A recent work of Panunzio 
[31] proved the validity of the arc-length-ratio method for propagating uncertainties in systems with internal resonance. The problem of internal resonance is out of the scope of the proposed paper (due to the studied structure), and further work should be achieved in order to see how the phase criterion copes with internal resonance.

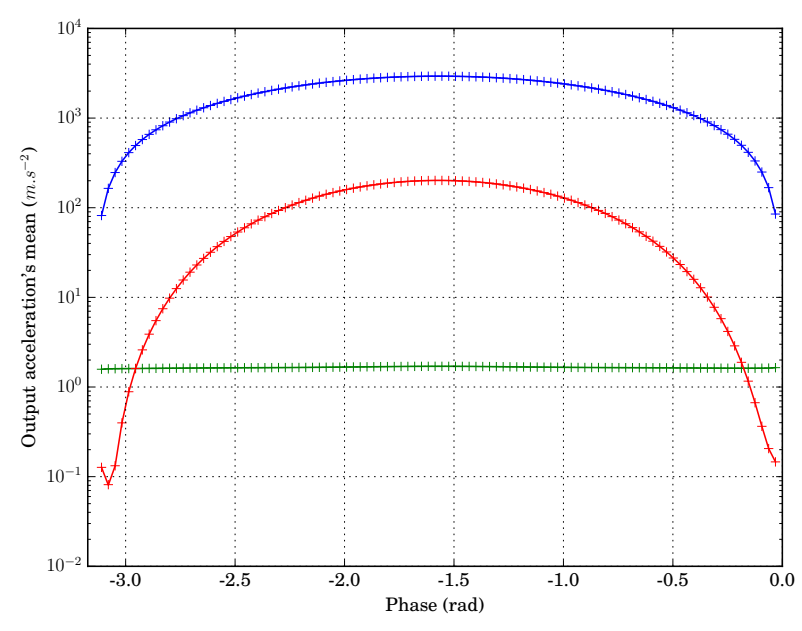

(a)

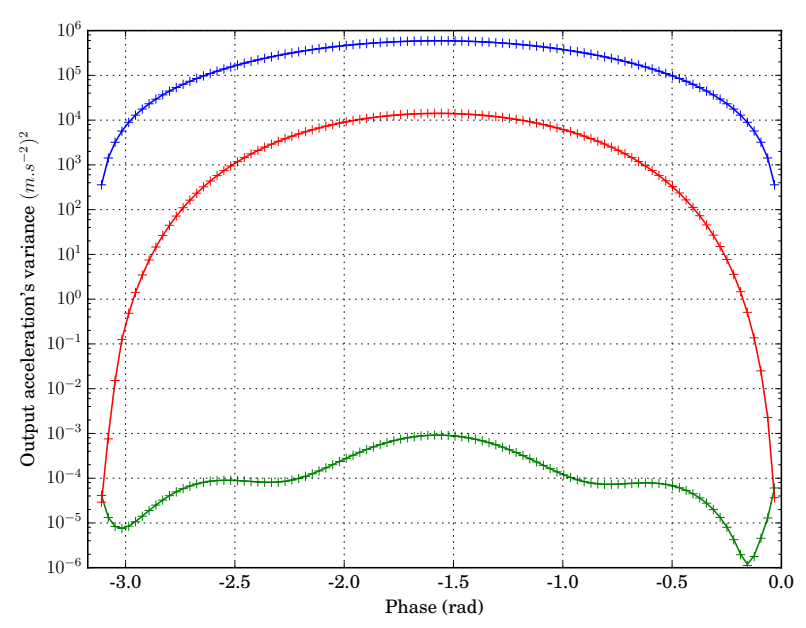

(c)

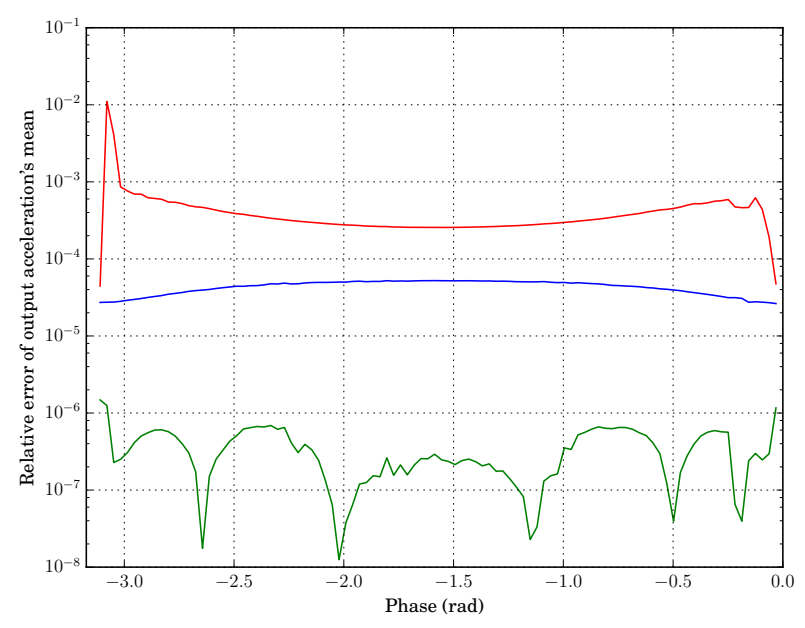

(b)

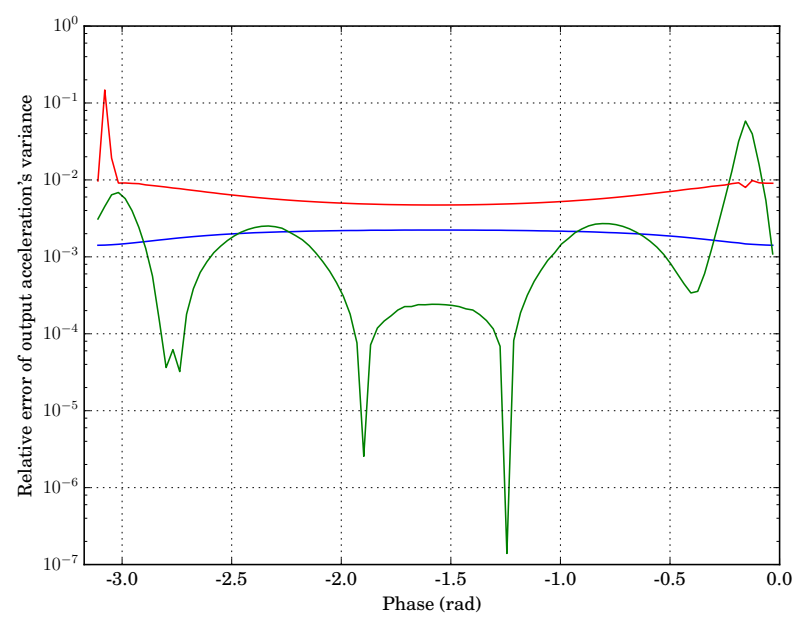

(d)

Figure 6: Mean (a), relative error of the mean (b), variance (c) and relative error of the variance (d) for the stochastic amplitude response. The color code is the same as that in Fig. 2.

\subsubsection{Impact on the usual indicators}

For stochastic analysis, engineers usually rely on indicators such as the mean, the standard deviation or the percentiles. These notions enable the engineer to have a quick understanding of the stochastic solution by going back to deterministic coefficients. The mean provides the average response while the standard deviation estimates its scatter and can also be used to provide an estimation of the envelope of the response. When using a phase criterion instead of a frequency criterion, these indicators can no longer be used a priori: the frequency is a stochastic unknown of the problem along with the amplitude. A first method to retrieve the initial meaning of the indicators is to compare the mean/variance/percentiles of the amplitude to the frequency mean, which is a deterministic notion. On the other hand, the standard deviation cannot be used to provide the lower and upper limits of the envelope. This problem is triggered by the return points in the solution and is not dependent on the phase criterion that we developed: the same problem would arise with an arc-length-ratio criterion. This puts into perspective the relevance of the usual indicators for the engineer. The mean is still a quality tool to estimate the average response. The variance can still estimate a scatter, but it can no longer be used for an estimation of the envelope, along with the percentiles. In order to obtain a good approximation of the envelope though, the engineer has other tools at his or her disposal, such as the Delaunay Triangulation [32], however the cost of such a technique is high and requires additional coding. 


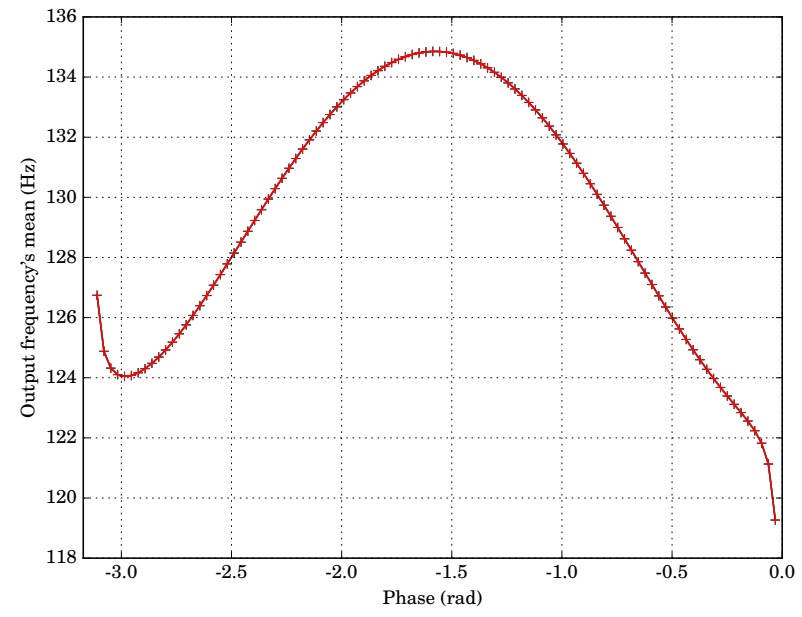

(a)

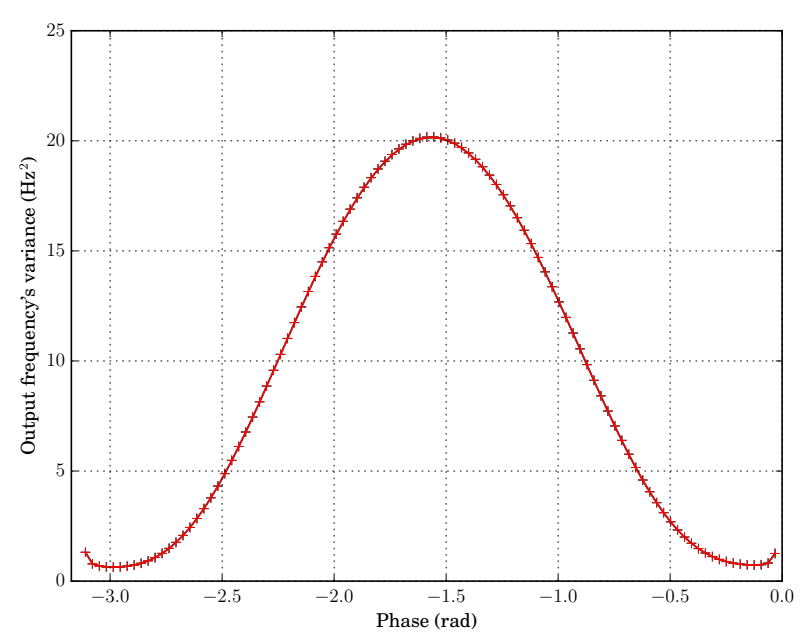

(c)

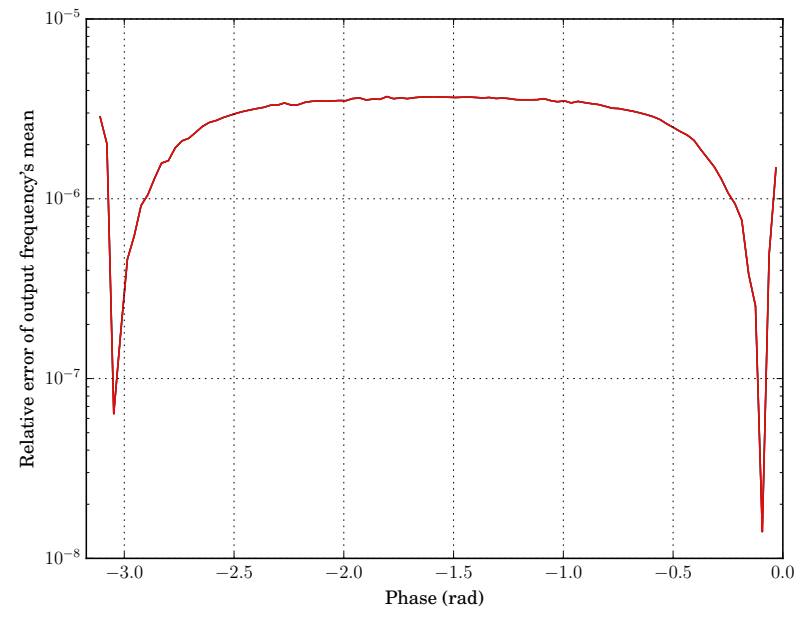

(b)

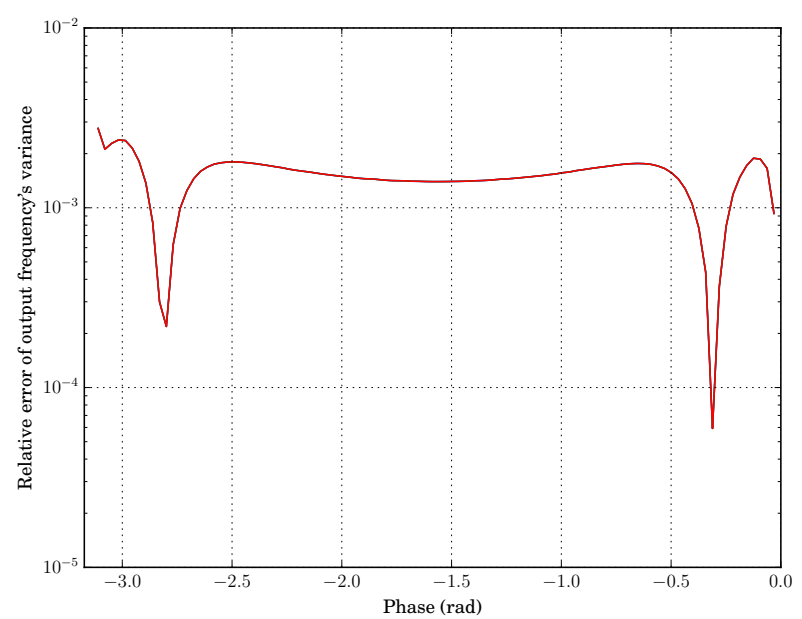

(d)

Figure 7: Mean (a), relative error of the mean (b), variance (c) and relative error of the variance (d) for the stochastic frequency response. The color code is the same as that in Fig. 2.

\section{Results}

In this section we calculate the stochastic response using the proposed methodology, starting from the updating of the model to quantify the uncertainties, and using a polynomial chaos appropriate for the HBM in order to obtain the envelope and the mean of the numerical response. The latter will be compared to the experiments.

\subsection{Updating of the model}

Figure 5 shows the validity of the calculation compared to the results of the experiments. The comparison is shown for different excitation levels but only for the fundamental Harmonic. We observe that the resonance frequency of the response is increasing along with the excitation level. These results validate the model developed in Equation (1) which takes into account the non-ideal boundary conditions. However, the updating of two independent parameters (the half effective length $l$ and the damping coefficient $\mu$ ) was required for each excitation level. The modifications made to the variables are presented in Table 3. This need of model updating for each excitation leads to the study of the propagation of uncertainties. Parameters $l$ and $\mu$ are then considered to be independent random variables of the nonlinear stochastic dynamic problem. We enforce that they follow a uniform law with lower and upper limits defined by the lower and upper values found in Table. 3 .

It is now possible to write the NIPCE for the clamped-clamped beam. The random variables of the dynamical system are $l$ and $\mu$, they both follow independent uniform laws, leading to a vector $\xi=\left(\xi_{l}, \xi_{\mu}\right)$ of uniform variables defined on $[-1,1]$ with density $\frac{1}{2}$. The use of uniform laws leads to the use of Legendre polynomials [33]. The uncertainties that we use do not have a physical background, they are calculated empirically to envelope the output response curves. All of the other variables of the system are deterministic, 


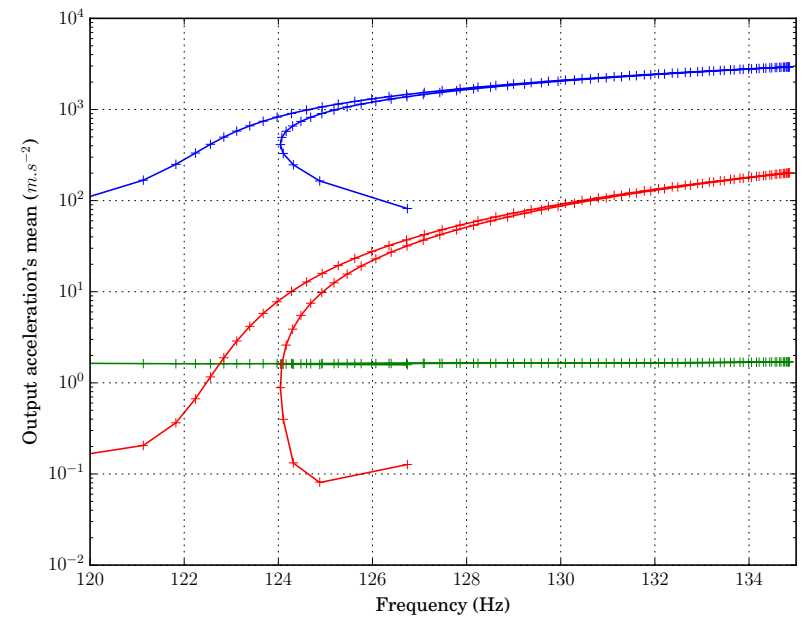

(a)

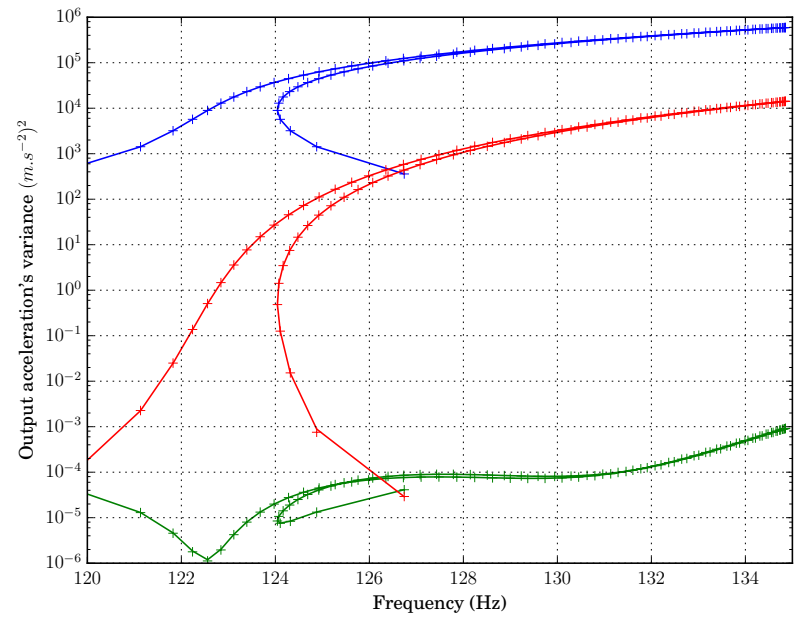

(b)

Figure 8: Mean (a) and variance (b) of the stochastic response for MC (solid lines) and PCE (crosses) simulations. The color code is the same as that in Fig. 2.

yet the phase plays a special role in the calculation of the NIPCE, so we write $x=\Phi$ where $\Phi$ is the phase. For a given calculation of the HBM, the phase is calculated through linear interpolation of the phase at the frequencies provided by the HBM. This does not require any change in the deterministic code, but rather a small addendum of post-calculation. The main goal of this study is to use empirical uncertainties in order to embed the experimental responses in the numerical envelopes generated through the PCE. The stochastic moments (mean, variance) will be calculated to validate the PCE by comparison with MonteCarlo simulations, and then the boundaries of the envelope will be compared to the experiments to validate the method.

\subsection{Numerical validation}

Firstly, we aim for the convergence of the PCE coefficients by increasing the order of the polynomial chaos. To do so, we study the mean and the variance of the stochastic response of the Duffing oscillator with increasing polynomial order (from 1 up to 5). It was demonstrated that convergence is reached at the order $p=4$ (results not presented here with the objective of concision). The polynomial chaos order will be set to 4 for the rest of the study.

Once convergence is obtained, we need to validate the precision of the method. The results are compared to a converged Monte-Carlo simulation, the solutions generated through PCE and Monte-Carlo simulations are obtained with the same 1000 random samples in the experiment plan. The average response (mean value) and the variance are indicated on Figure 6 and in Figure 7.

A relative error lower than $0.1 \%$ for the first two stochastic moments is observed, which validates the PCE approach. The absolute mean and variance are plotted in Figures $6 \mathrm{a}$ and $7 \mathrm{a}$ for the mean and Figures $6 \mathrm{c}$ and $7 \mathrm{c}$ for the variance, while the relative errors are plotted in Figures $6 \mathrm{~b}$ and $7 \mathrm{~b}$ for the mean and Figures $6 \mathrm{~d}$ and $7 \mathrm{~d}$ for the variance. For absolute responses, the full lines are the Monte-Carlo results while the crosses are the PCE results. The convergence analysis was carried out for both the amplitude and the frequency, for the entire phase range. The PCE with the phase criterion shows no sign of spurious oscillations, validating the hypothesis made in Section 4.2.3 that there is no need to control the critical points in a phase criterion approach of the PCE. Now that convergence has been achieved,the stochastic amplitude response (mean and variance) can be plotted with the mean of the frequency as a x-axis, as discussed in Section 4.2.4. Figure 8 demonstrates the capability of returning to the use of classical indicators. The mean and variance of the amplitude response compared to the mean of the frequency allow the notion of hardening effect at the resonance to be preserved. Moreover, high variances are found for the fundamental and the third Harmonics, which are affected by the dynamics of the system, while the variance of the second Harmonic is low because it is mostly due to the input excitation on the second Harmonic.

\subsection{Experimental validation}

The converged PCE has been validated by a comparison with Monte-Carlo Simulations. We first build a PCE for a numerical excitation of $4 \mathrm{~m}_{\mathrm{s}} \mathrm{s}^{-2}$. This excitation is generated through the experimental input extraction detailed in Section 3.2. Thanks to the PCE we generate the numerical $5 \%$ and $95 \%$ percentiles for the three Harmonics of the stochastic solution. Figure 9 shows these envelopes along with the experimental 


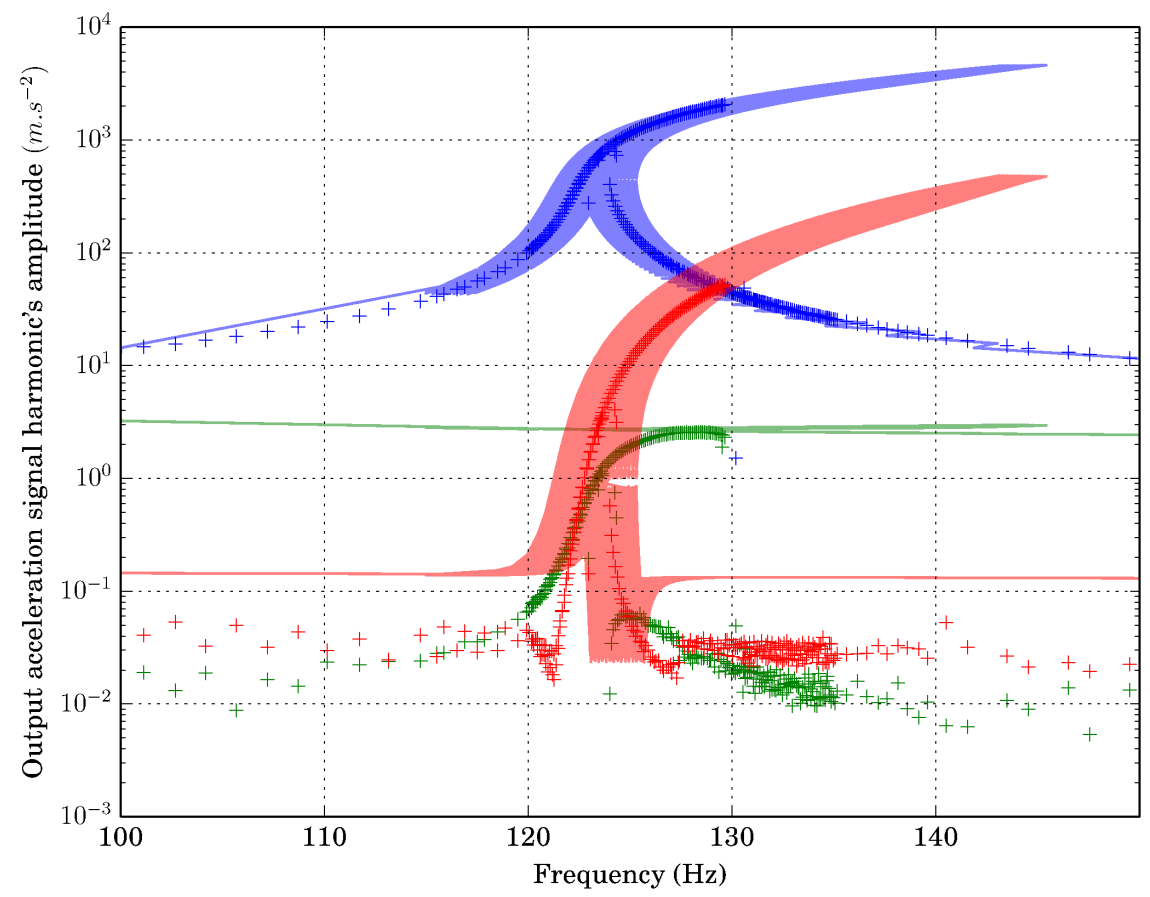

Figure 9: Experimental (crosses) and envelope (blurred) results for Harmonics 1 (blue), 2 (green) and 3 (red).

response. The fundamental Harmonic of the experimental response (blue line) is fully embedded in the blue envelope which validates the predictive approach of polynomial chaos. Regarding the second and third Harmonics respectively in green and red, the numerical envelopes are not fully wrapped around the experimental Harmonics, yet they provide a conservative prediction for the engineer, since the envelopes are above the experimental responses. The discrepancies between the calculations and the experiments do not come from polynomial chaos, but rather from the input excitation model as discussed in Section 3.2. The third Harmonic mostly maintains a good response around the resonance, but the second Harmonic is irrelevant over the entire frequency domain. Further research should be made in order to understand the physical meaning of this second harmonic, and to implement it in a Duffing model, by adding a quadratic term for instance. Once the method has been validated through a comparison with a single experiment, we can easily reproduce the calculations for various excitation levels, using the same procedure. Figure 10 shows the stochastic response of the fundamental for excitation levels of 1 (blue) and 8 (red) $m . s^{-2}$. Figure 10 is therefore the stochastic version of Figure 5. The main improvement is that this stochastic prediction did not require any updating. It can be noted that the experiments are never really centered on the stochastic envelopes. This is because the envelope is centered around the stochastic mean (dotted line) while the quantification of uncertainties only ensures that the experiments will be inside the envelopes. Moreover, it seems that the size of the envelope increases for high excitation levels. The uncertain parameters have a more influential role at high levels of excitations, when the nonlinearity is the most active.

\section{Conclusion}

This paper studies the stochastic non-linear dynamic response of an excited clamped-clamped beam. Non-intrusive polynomial chaos has been implemented in order to compute the stochastic response with great time efficiency compared to that of Monte-Carlo simulations.

The introduced technique is a non-intrusive PCE combining the Harmonic Balance Method and a MoorePenrose corrector. To overcome the main difficulty, which is the presence of multiple solutions for a given frequency due to the return points of the Duffing oscillator, a deterministic phase criterion is implemented. The phase is strictly decreasing over the frequency range, which gives a meaning to the computation of a PCE for a discrete set of phases.

The PCE is firstly compared to Monte-Carlo simulations, showing an excellent agreement while dramatically reducing the calculation time. Secondly, the PCE is compared to the experiments and shows a capacity for predicting the envelope of an experimental response. 


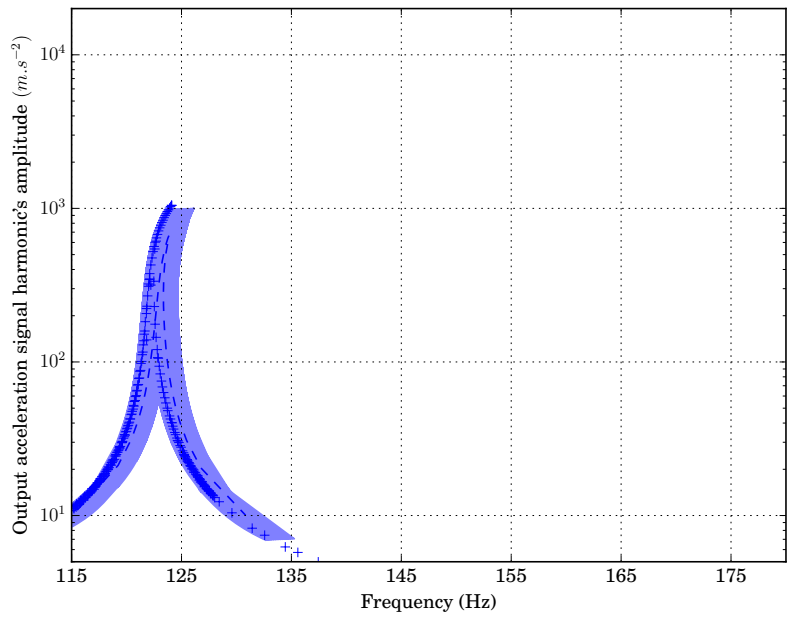

(a)

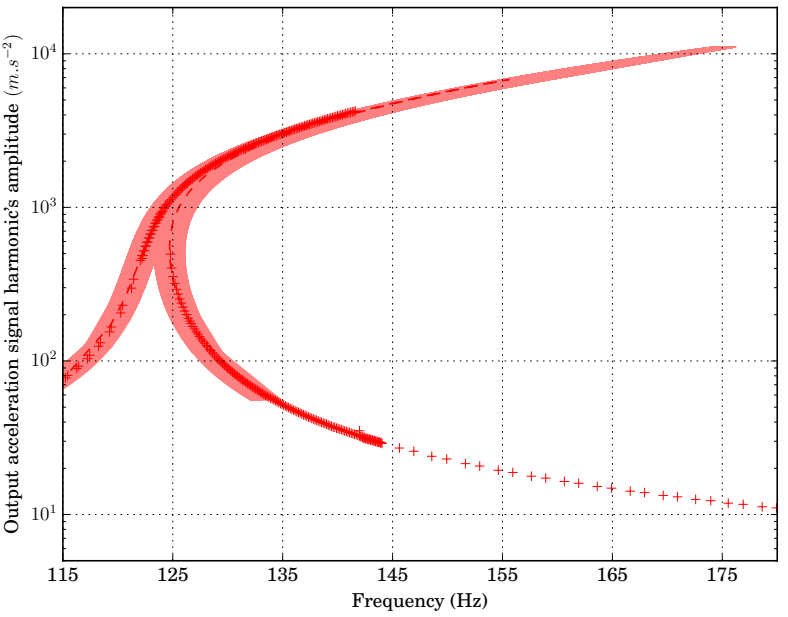

(b)

Figure 10: Experimental response (crosses), numerical mean (dotted line) and envelope (blurred) for the first Harmonic with excitation levels of 1 (blue) and 8 (red) $m \cdot s^{-2}$.

\section{Acknowledgement}

\section{J.-J. Sinou acknowledges the support of the Institut Universitaire de France.}

\section{References}

[1] A. Nayfeh and D. Mook, Nonlinear Oscillations. John Wiley \& Sons, New York, 1979.

[2] A. Vakakis, L. Manevitch, Y. Mikhlin, V. Pilipchuk, and A. Zevin, Normal Modes and Localization in Nonlinear Systems. John Wiley \& Sons, 1996.

[3] G. Kerschen, M. Peeters, J.-C. Golinval, and A. Vakakis, "Nonlinear normal modes, part 1: a useful framework for the structural dynamicist," Mech. Syst. Signal Process., vol. 23, pp. 170-194, 2009.

[4] E. Sarrouy and J.-J. Sinou, Non-linear periodic and quasi-periodic vibrations in mechanical systems - on the use of the harmonic balance methods. INTECH Open Access Publisher, 2011.

[5] M. Claeys, J.-J. Sinou, J.-P. Lambelin, and B. Alcoverro, "Multi-harmonic measurements and numerical simulations of nonlinear vibrations of a beam with non-ideal boundary conditions," Communications in Nonlinear Science and Numerical Simulation, vol. 19, no. 12, pp. 4196-4212, 2014.

[6] J. K. Kevorkian and J. D. Cole, Multiple scale and singular perturbation methods, vol. 114. Springer Science \& Business Media, 2012.

[7] N. Metropolis, A. Rosenbluth, M. Rosenbluth, A. Teller, and E. Teller, "Equation of state calculations by fast computing machines," Chem. Phys, vol. 21, p. 1087, 1953.

[8] R. Ghanem and P. Spanos, Stochastic finite elements : a spectral approach. Springer-Verlag, 1991.

[9] E. Sarrouy, E. Pagnacco, and E. Souza De Cursi, "A constant phase approach for the frequency response of stochastic linear oscillators," Mechanics and Industry, vol. 17, p. 206, Feb. 2016.

[10] E. Sarrouy, "Étude d'un oscillateur de duffing stochastique via la phase," in Proceedings du 13e Colloque National en Calcul des Structures, 15-19 mai 2017, Giens, France, 2017.

[11] N. Hansen and A. Ostermeier, "Completely derandomized self-adaptation in evolution strategies," Evol Comput, vol. 9, no. 2, pp. 159-195, 2001.

[12] A. Nayfeh, "Nonlinear transverse vibrations of beams with properties that vary along the length," Journal of the Acoustical Society of America, vol. 53, no. 3, pp. 766-770, 1973.

[13] T. Cameron and J. Griffin, "An alternating frequency time domain method for calculating the steady state response of nonlinear dynamic systems," Journal of Applied Mechanics, vol. 56, pp. 149-154, 1989.

[14] V. Jaumouillé, J.-J. Sinou, and B. Petitjean, "An adaptive harmonic balance method for predicting the nonlinear dynamic responses of mechanical systems. Application to bolted structures," Journal of Sound and Vibration, vol. 329, pp. 40484067, 2010.

[15] R. Penrose, "A generalized inverse for matrices," Mathematical proceedings of the Cambridge philosophical society, vol. 51, no. 3, pp. 406-413, 1955.

[16] J. Didier, J.-J. Sinou, and B. Faverjon, "Nonlinear vibrations of a mechanical system with non-regular nonlinearities and uncertainties," Communications in Nonlinear Science and Numerical Simulation, vol. 18, no. 11, pp. 3250-3270, 2013.

[17] J.-J. Sinou, J. Didier, and B. Faverjon, "Stochastic nonlinear response of a flexible rotor with local nonlinearities," International Journal of Non-Linear Mechanics, vol. 74, pp. 92-99, 2015.

[18] S. Hosder, R.-W. Walters, and M. Balch, "Efficient Sampling for Non-Intrusive Polynomial Chaos Applications with Multiple Input Uncertain Variables," $9^{t h}$ AIAA Non-Deterministic Approaches Conference, 2007.

[19] B. Debusschere, H. Najm, P. Pebay, O. Knio, R. Ghanem, and O. Maitre, "Numerical Challenges in the use of Polynomial Chaos Representations for Stochastic Processes," SIAM Journal on Scientific Computing, vol. 26, no. 2, pp. 698-719, 2004.

[20] D. Xiu and G. Karniadakis, "Modeling Uncertainty in Flow Simulations via Generalized Polynomial Chaos," Journal of Computational Physics, vol. 187, no. 1, pp. 137-167, 2003.

[21] R. Ghanem and R. Kruger, "Numerical solution of spectral stochastic finite element systems," Computer Methods in Applied Mechanics and Engineering, vol. 129, no. 3, pp. 289-303, 1996. 
[22] O. Le Maître and O. M. Knio, Spectral methods for uncertainty quantification: with applications to computational fluid dynamics. Springer Science \& Business Media, 2010.

[23] N. Wiener, "The homogeneous chaos," American Journal of Mathematics, vol. 60, no. 4, pp. 897-936, 1938.

[24] R. W. Walters, "Towards stochastic fluid mechanics via polynomial chaos," in Proceedings of the 41st AIAA Aerospace Sciences Meeting and Exhibit, number AIAA-2003-0413, Reno, NV, vol. 88, 2003.

[25] S. Hosder, R. Perez, and R. Walters, "A non-intrusive polynomial chaos method for uncertainty propagation in cfd simulations," Faculty Research \& Creative Works, vol. 2020, 2006.

[26] S. Hosder and R. W. Walters, "Non-intrusive polynomial chaos methods for uncertainty quantification in fluid dynamics," in 48th AIAA Aerospace Sciences Meeting, Jan, pp. 4-7, 2010.

[27] J. Helton and F. Davis, "Latin Hypercube Sampling and the propagation of uncertainty in analysis of complexe systems," Reliability Engineering and Sytem Safety, vol. 81, pp. 23-69, 2003.

[28] E. Jacquelin, S. Adhikari, J.-J. Sinou, and M. I. Friswell, "Polynomial chaos expansion and steady-state response of a class of random dynamical systems," Journal of Engineering Mechanics, vol. 141, no. 4, 2014.

[29] A. Panunzio, L. Salles, and C. Schwingshackl, "Uncertainty propagation for nonlinear vibrations: A non-intrusive approach," Journal of Sound and Vibration, vol. 389, pp. 309 - 325, 2017.

[30] E. H. Moussi, S. Bellizzi, B. Cochelin, and I. Nistor, "Nonlinear normal modes of a two degrees-of-freedom piecewise linear system," Mechanical Systems and Signal Processing, vol. 64, pp. 266-281, 2015.

[31] A. Panunzio, L. Salles, C. Schwingshackl, and M. Gola, "Asymptotic numerical method and polynomial chaos expansion for the study of stochastic non-linear normal modes," vol. 7B: Structures and Dynamics, ASME Turbo Expo 2015: Turbine Technical Conference and Exposition, 2015.

[32] J. R. Shewchuk, "Delaunay refinement algorithms for triangular mesh generation," Computational Geometry, vol. 22, no. 1, pp. 21 - 74, 2002. 16th ACM Symposium on Computational Geometry.

[33] M. Eldred, C. Webster, and P. Constantine, "Evaluation of Non-Intrusive Approaches for Wiener-Askey Generalized Polynomial Chaos," $49^{\text {th }}$ AIAA Structures, Structual Dynamics, and Materials Conference, 2008. 